\title{
BeppoSAX observation of a large long-duration $X$-ray flare from UX Arietis
}

\author{
E. Franciosini ${ }^{1}$, R. Pallavicini ${ }^{1}$, and G. Tagliaferri ${ }^{2}$ \\ 1 Osservatorio Astronomico di Palermo, Piazza del Parlamento 1, 90134 Palermo, Italy \\ e-mail: francio@oapa.astropa.unipa.it, pallavic@oapa.astropa.unipa.it \\ 2 Osservatorio Astronomico di Brera, Via E. Bianchi 46, 23807 Merate, Italy \\ e-mail: gtagliaf@merate.mi.astro.it
}

Received 10 April 2001 / Accepted 7 June 2001

\begin{abstract}
We present two X-ray observations of the RS CVn binary system UX Arietis performed with BeppoSAX in August 1997 and August 1998. A large flare lasting for about 1 day was detected during the first observation, while the star was quiescent during the second one. Hard X-ray emission up to $50 \mathrm{keV}$ was detected in the PDS instrument during the rise and at the peak of the flare. Time-resolved spectroscopy of the flare with the LECS and MECS detectors shows that temperatures as high as $~ 100 \mathrm{MK}$ were reached at the flare peak; such high temperatures are responsible for the high-energy tail seen in the PDS. We confirm that the coronal abundance of UX Ari is strongly subsolar $\left(Z / Z_{\odot} \simeq 0.2-0.3\right)$; no significant variation of the metallicity is observed during the flare. We show that the new LECS response matrices released in January 2000 seem to solve the problem of the high hydrogen column densities systematically found in previous BeppoSAX observations of stellar coronae. We find in fact for the quiescent spectrum $N_{\mathrm{H}}=2.6 \times 10^{19} \mathrm{~cm}^{-2}$, compatible with the expected value; during the flare $N_{\mathrm{H}}$ is higher by a factor 5 , probably due to the ejection of material. The flare evolution has been analysed using the two-ribbon flare model by Poletto et al. (1988), finding a very good agreement between the model and the observations.
\end{abstract}

Key words. binaries: close - stars: activity - stars: coronae - stars: individual: UX Ari - X-rays: stars

\section{Introduction}

UX Ari is one of the most active RS CVn binary systems, consisting of a G5V and a K0IV star, with an orbital period of 6.44 days and an inclination of $60^{\circ}$; it is located at a distance of 50.2 pc (from Hipparcos, ESA 1997). This system shows an intense coronal emission at X-ray wavelengths, and thus it has been extensively studied since its discovery as an X-ray source by the HEAO-1 satellite (Walter et al. 1978). During the ROSAT All Sky Survey UX Ari was detected at the quiescent level, with an X-ray luminosity $L_{\mathrm{X}} \sim 1.2 \times 10^{31} \mathrm{erg} \mathrm{s}^{-1}$ in the $0.1-2.4 \mathrm{keV}$ band (Dempsey et al. 1993a); the spectrum was reproduced using a two-component optically-thin plasma model with temperatures of 2 and $16 \mathrm{MK}$, emission measure ratio $E M_{\text {hot }} / E M_{\text {cool }} \sim 4$ and a hydrogen column density of $1.2 \times 10^{19} \mathrm{~cm}^{-2}$ (Dempsey et al. 1993b).

An intense long-lasting X-ray flare was observed by Tsuru et al. (1989) with the Ginga satellite. The flare had a peak luminosity of $2 \times 10^{32} \mathrm{erg} \mathrm{s}^{-1}$ in the 2 $20 \mathrm{keV}$ band, and decayed with a timescale of $\sim 12$ hours. The total energy released during the flare was $\gtrsim 10^{37} \mathrm{erg}$.

Send offprint requests to: E. Franciosini, e-mail: francio@oapa.astropa.unipa.it
Spectral analysis yielded a peak temperature $T \sim 80 \mathrm{MK}$ and an emission measure $E M \sim 10^{55} \mathrm{~cm}^{-3}$. The $6.7 \mathrm{keV}$ iron line resulted significantly weaker than expected for a solar abundance plasma, suggesting a lower than solar metallicity for the corona of UX Ari.

Güdel et al. (1999) observed UX Ari with $A S C A$ for 14 hours. The star was in a steady quiescent state for most of the observation, and then underwent a strong flare, of which only the rise phase and the peak were observed. The rise phase lasted for $\sim 2$ hours, during which the X-ray emission increased by a factor $\sim 10$, reaching a peak $L_{\mathrm{X}} \sim 1.3 \times 10^{32} \mathrm{erg} \mathrm{s}^{-1}$ in the $0.4-10 \mathrm{keV}$ band. At the flare peak temperatures in excess of $100 \mathrm{MK}$ were derived from the spectral analysis. The spectral fits required subsolar abundances, which increased significantly during the flare rise; in particular the $\mathrm{Fe}$ abundance increased from 0.17 solar during quiescence to 0.89 solar at the flare peak.

In this paper we present the results of two BeppoSAX observations of UX Ari. In the first observation, the star was caught during a long-duration strong flare, which was observed from the final part of the rise phase to the end of the decay; in addition, hard X-rays $(\sim 15-50 \mathrm{keV})$ were detected by the PDS instrument. Observations and data 
analysis are described in Sect. 2; in Sect. 3 we present the results, and in Sect. 4 we analyse the flare evolution using a two-ribbon flare model. Discussion and conclusions are given in Sect. 5 .

\section{Observations and data analysis}

UX Ari has been observed twice by BeppoSAX using the LECS (0.1-10 keV), the two available MECS (1.6-10 keV) and the PDS (15-300 keV) instruments. The first observation started at 21:44 UT on August 28, 1997 and ended at 01:04 UT on August 30, 1997, after 27 hours of elapsed time. The star was observed again exactly one year later for 59 hours, from 18:23 UT on August 28, 1998 to 05:24 UT on August 31, 1998. During the second observation, simultaneous radio observations were performed at the VLA; these data will be discussed in a forthcoming paper (Franciosini et al., in preparation; a preliminary analysis is presented in Franciosini et al. 2001). The effective exposure times for the LECS, MECS and PDS instruments were respectively 22,48 and $45 \mathrm{ksec}$ for the first observation, and 44, 107 and $98 \mathrm{ksec}$ for the second one. The lower exposure time in the LECS is due to the fact that this instrument was operated only in Earth shadow, thus reducing its observing efficiency.

The LECS and MECS data analysis was based on the linearized and cleaned event files obtained from the BeppoSAX Science Data Center (SDC) on-line archive. Spectra and light curves were accumulated using the XSELECT v.1.4b software in the FTOOLS v4.2 package, using an extraction region of 8 and 4 arcmin radius for the LECS and MECS, respectively; the chosen regions provide more than $90 \%$ of the source counts at all energies for both instruments. LECS and MECS background spectra accumulated from blank field exposures and available at the SDC were used for spectral analysis. For the PDS, both the light curve and the spectrum were retrieved from the SDC on-line archive. The PDS spectrum obtained by the standard pipeline is already background-subtracted and thus no further correction is necessary.

The spectral analysis was performed with the XSPEC v11.00 package, using the response matrices released by the SDC in September 1997 for the MECS and PDS, while for the LECS the new response matrices released in January 2000 have been used. The spectra were rebinned in order to have at least 50 counts per bin. The spectral fits were performed using $\chi^{2}$ statistics with weights evaluated according to Gehrels (1986).

\section{Results}

In Figs. 1 and 2 we show the light curves of UX Ari observed by the LECS, MECS and PDS instruments. During the Aug. 1997 observation, a large long-lasting flare was detected, showing a similar evolution in both the LECS and MECS instruments. The flare started before the beginning of the BeppoSAX observation, and only the final part of the rise phase was observed for $\sim 2$ hours.

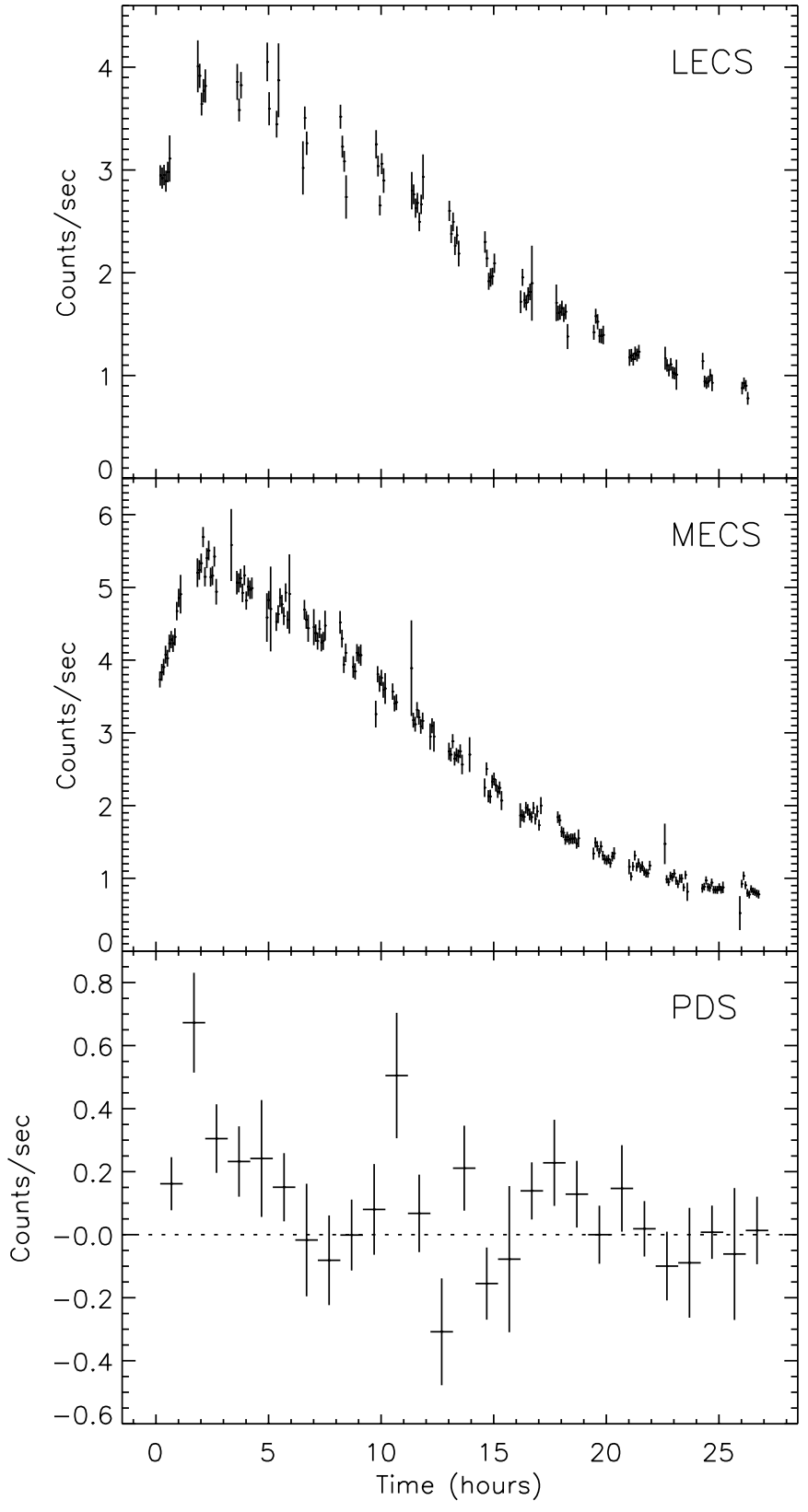

Fig. 1. UX Ari light curves observed on August 28-30, 1997 by the LECS (top panel), MECS (middle panel) and PDS (bottom panel) instruments. LECS and MECS data have been binned over $300 \mathrm{~s}$, while PDS data are binned over $3600 \mathrm{~s}$. Time is in hours (UT) from the beginning of the observation.

The X-ray emission then decayed slowly, returning to a presumably quiescent level at the end of the observation; the $1 / e$ decay time in the MECS was $\sim 13$ hours. The count rate at the peak was a factor $\sim 8$ higher than at the end of the flare. Weak PDS emission was detected during the rise phase and at the peak of the flare.

In Aug. 1998 the star was at a quiescent level, showing only small variations around a mean count rate of $\sim 0.40 \mathrm{cts} \mathrm{s}^{-1}$ in the LECS and $\sim 0.27 \mathrm{cts} \mathrm{s}^{-1}$ in the MECS, i.e. a factor $\sim 3$ lower than that observed at the end of the 


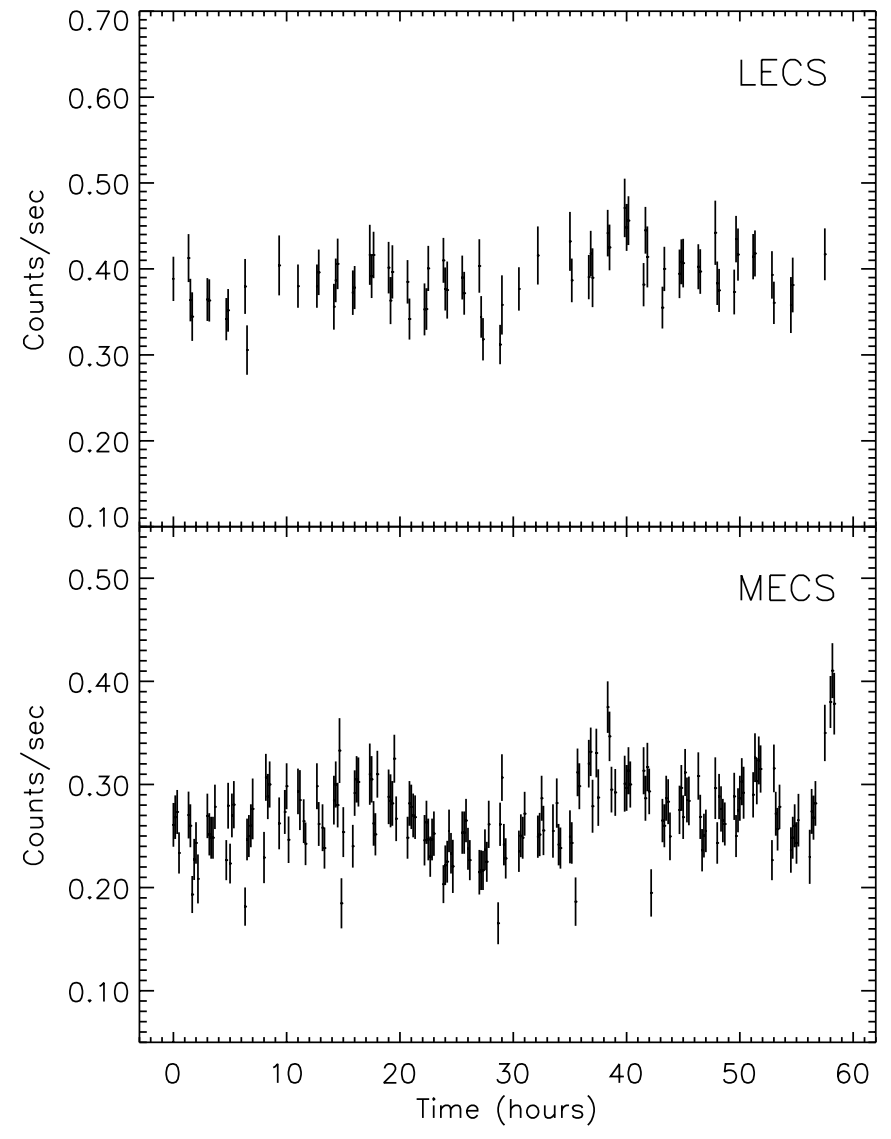

Fig. 2. LECS (top panel) and MECS (bottom panel) light curves for the August 28-31, 1998 observation. Data have been binned over $600 \mathrm{~s}$. The emission level in the LECS and MECS is a factor $\sim 3$ lower than at the end of the Aug. 1997 flare. Time is in hours (UT) from the beginning of the observation.

Aug. 97 flare. The scatter ( $1 \sigma$ standard deviation) around the mean is $\sim 10 \%$ and $\sim 13 \%$ for the LECS and MECS, respectively. Given that the data cover only a fraction of 0.4 of the orbital period, we cannot determine whether a contribution due to rotational modulation is present, or if the observed variations are entirely due to intrinsic variability of the X-ray emission. No significant emission is detected in the PDS: the count rate oscillates around zero with a standard deviation of $\sim 0.2 \mathrm{cts} \mathrm{s}^{-1}$.

\subsection{Spectral analysis of the LECS and MECS data}

During the first observation the count rate is high enough to allow time-resolved spectroscopy of the flare decay using both the LECS and the MECS. We selected three time intervals at the top, in the middle of the decay, and at the end of the flare. The integration times have been chosen in order to have at least 5000 counts in the LECS and 10000 counts in the two combined MECS detectors. The three intervals have a total duration (including data gaps) of $\sim 4.5,5.5$ and 6 hours, respectively. For the Aug. 1998 data a spectrum for the full observation was considered.
All spectra have been fitted assuming an optically-thin thermal plasma with two discrete temperature components, using the MEKAL model emissivities (Mewe et al. 1995) with variable global metal abundances, and taking into account the effect of interstellar absorption. The interstellar column density $N_{\mathrm{H}}$ was left as a free parameter.

When fitting LECS and MECS spectra together, a relative normalization factor must be included to account for the uncertainties in the intercalibration of the instruments. This factor was left free to vary, finding best-fit values between 0.78 and 0.82 , in agreement with the allowed range of $0.7-1$ (Fiore et al. 1999).

Figure 3 shows the four LECS and MECS spectra together with the best-fit models; the best-fit parameters are given in Table 1. At the flare peak temperatures of $2.9 \mathrm{keV}$ and $9.5 \mathrm{keV}$ are found; the emission measure ratio is $E M_{2} / E M_{1} \sim 1$ and the metallicity is $Z=0.3 Z_{\odot}$. At the end of the flare the temperatures have decreased to 0.9 and $2.6 \mathrm{keV}$, while $E M_{2} / E M_{1} \sim 3$ and $Z=0.2 Z_{\odot}$. In Aug. 1998 we find temperatures of 0.9 and $2.2 \mathrm{keV}$, $Z=0.25 Z_{\odot}$ and $E M_{2} / E M_{1} \sim 2$. The quiescent parameters are in good agreement with those obtained by Güdel et al. (1999) during the $A S C A$ observation. We also note that the spectral parameters derived at the end of the Aug. 1997 flare, apart from the higher emission level, are very similar to those obtained for the quiescent emission; it is therefore likely that at the end of the Aug. 1997 observation the star had returned to a quiescent state.

The best-fit value of the hydrogen column density $N_{\mathrm{H}}$ resulted equal to $\sim 1 \times 10^{20} \mathrm{~cm}^{-2}$ during the whole Aug. 1997 flare and to $2.6 \times 10^{19} \mathrm{~cm}^{-2}$ for the Aug. 1998 quiescent emission. The latter value is in very good agreement with the value $N_{\mathrm{H}}=1.2 \times 10^{19} \mathrm{~cm}^{-2}$ obtained by Dempsey et al. (1993b) by fitting the ROSAT All-Sky Survey data, and also with the expected $N_{\mathrm{H}} \sim 10^{19} \mathrm{~cm}^{-2}$ derived assuming an interstellar hydrogen volume density of $0.07 \mathrm{~cm}^{-3}$ (Paresce 1984) given the distance of UX Ari. We note that if we had used the old (Sep. 1997) LECS response matrices, we would have obtained for the quiescent spectrum $N_{\mathrm{H}}=8 \times 10^{19}$, i.e. nearly one order of magnitude higher than expected, similar to what was found in all previous BeppoSAX observations of stellar coronae (Favata et al. 1997; Favata \& Schmitt 1999; Rodonò et al. 1999; Tagliaferri et al. 1999; Covino et al. 2000; Maggio et al. 2000). Our result seems therefore to confirm the suggestion (Tagliaferri et al. 1999) that the high $N_{\mathrm{H}}$ systematically required to fit previous BeppoSAX stellar spectra was most likely due to calibration problems of the LECS detector at low energies. Note however that, even with the new matrices, the hydrogen column density derived during the flare is still high (although lower by a factor $\sim 1.4$ than the one obtained with the old matrices).

\subsection{The hard $X$-ray spectrum}

Figure 4 shows the total LECS+MECS+PDS spectrum of the Aug. 1997 flare, integrated over the first $24 \mathrm{ksec}$ of the 
Table 1. Best-fit parameters of the two-temperature MEKAL models. Errors are computed at the $90 \%$ confidence levels for four interesting parameters.

\begin{tabular}{|c|c|c|c|c|c|c|c|c|c|c|}
\hline & Interval & $\begin{array}{r}N_{\mathrm{H}} / 10^{19} \\
\mathrm{~cm}^{-2}\end{array}$ & $Z / Z_{\odot}$ & $\begin{array}{c}T_{1} \\
\mathrm{keV}\end{array}$ & $\begin{array}{c}T_{2} \\
\mathrm{keV}\end{array}$ & $\begin{array}{c}E M_{1} / 10^{53} \\
\mathrm{~cm}^{-3}\end{array}$ & $\begin{array}{c}E M_{2} / 10^{53} \\
\mathrm{~cm}^{-3}\end{array}$ & $\begin{array}{c}F_{x} / 10^{-10 a} \\
\text { erg } \mathrm{cm}^{-2} \mathrm{~s}^{-1}\end{array}$ & $\chi_{\mathrm{r}}^{2}$ & d.o.f. \\
\hline \multirow[t]{3}{*}{ Aug. 97} & peak & $10.7_{-3.7}^{+4.3}$ & $0.33_{-0.09}^{+0.08}$ & $2.89_{-0.28}^{+1.70}$ & $9.54_{-0.85}^{+2.58}$ & 57.4 & 52.7 & 6.2 & 0.99 & 258 \\
\hline & decay & $9.2_{-1.9}^{+2.7}$ & $0.18_{-0.04}^{+0.10}$ & $1.31_{-0.30}^{+0.14}$ & $4.00_{-0.12}^{+0.27}$ & 14.7 & 65.2 & 3.9 & 0.94 & 324 \\
\hline & end & $10.4_{-4.1}^{+5.1}$ & $0.19_{-0.09}^{+0.13}$ & $0.90_{-0.24}^{+0.32}$ & $2.63_{-0.23}^{+0.54}$ & 8.4 & 28.4 & 1.6 & 0.65 & 166 \\
\hline Aug. 98 & quiescent & $2.6_{-2.2}^{+2.6}$ & $0.25_{-0.08}^{+0.09}$ & $0.86_{-0.11}^{+0.17}$ & $2.17_{-0.14}^{+0.37}$ & 3.9 & 9.0 & 0.5 & 0.93 & 293 \\
\hline
\end{tabular}

${ }^{a}$ Unabsorbed X-ray fluxes in the $0.1-10 \mathrm{keV}$ band.
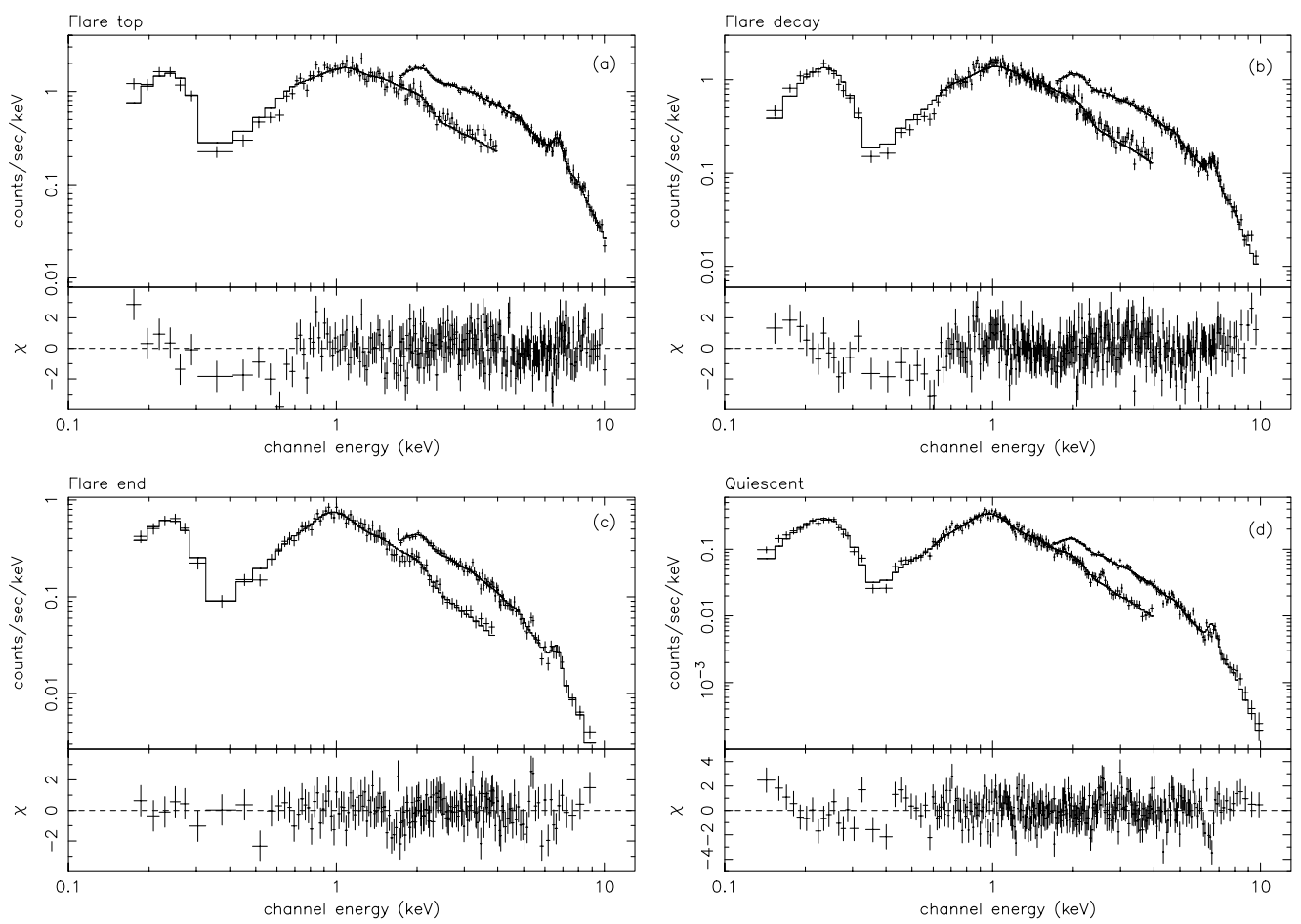

Fig. 3. Best-fits of the LECS+MECS spectra a) in Aug. 1997 at the flare peak, b) in the middle of the decay, c) at the end of the flare, and d) in Aug. 1998 for the entire observation.

observation, corresponding to the flare rise and peak where significant PDS emission was observed (cf. Fig. 1). The PDS spectrum retrieved from the SDC has been rescaled to account for the smaller integration time. Hard X-ray emission in the PDS is clearly detected up to at least 40 $50 \mathrm{keV}$. In the figure we also show the two-temperature thermal model that best fits the LECS and MECS data alone, extrapolated to the higher energies of the PDS instrument. The fit to the total spectrum has a reduced $\chi^{2}$ of 0.95 for 348 degrees of freedom, and it indicates that the hard X-ray emission can be attributed to the highenergy tail of the thermal emission from the hot flaring plasma. This result is consistent with those obtained for Algol (Favata \& Schmitt 1999) and AB Dor (Pallavicini 2001): in both cases the hard X-ray emission observed during large flares could be explained by thermal emission from the hot flaring plasma, with no need for any additional non-thermal component.

\subsection{Time evolution of the flare parameters}

In order to study the temporal evolution of the flare parameters only, we have fitted the MECS data using a onetemperature model, representing the flaring emission, plus a fixed two-temperature component with the parameters derived from the above analysis for the flare end. In this way it is possible to disentangle the contribution of the flaring plasma from the underlying quiescent emission. For this analysis we have considered only the MECS data, since this allows us to divide the dataset into smaller time intervals, still having enough counts for a good statistical analysis.

We have divided the dataset into 14 time intervals, chosen so as to have at least 7000 counts in each bin. The central time of each bin and the best-fit parameters are given in Table 2. The evolution with time of temperature, metallicity, emission measure and X-ray luminosity is shown in 
Table 2. Best-fit parameters of the one-temperature model plus the quiescent component for the MECS spectra. Errors are computed at the $90 \%$ confidence level for the three interesting parameters $Z / Z_{\odot}, T$ and $E M$.

\begin{tabular}{rrrcccccr}
\hline $\begin{array}{r}\text { Bin } \\
\text { no. }\end{array}$ & $\begin{array}{c}t_{\text {bin }}^{a} \\
\text { hours }\end{array}$ & $Z / Z_{\odot}$ & $\begin{array}{c}T \\
\mathrm{keV}\end{array}$ & $\begin{array}{c}E M / 10^{54} \\
\mathrm{~cm}^{-3}\end{array}$ & $\chi_{\mathrm{r}}^{2}$ & dof & $\begin{array}{c}F_{\mathrm{x}} / 10^{-10 b} \\
\mathrm{erg} \mathrm{cm}^{-2} \mathrm{~s}^{-1}\end{array}$ & $\begin{array}{r}L_{\mathrm{X}} / 10^{31 b} \\
\mathrm{erg} \mathrm{s}^{-1}\end{array}$ \\
\hline 1 & 0.24 & $0.25_{-0.25}^{+0.26}$ & $8.82_{-1.78}^{+2.76}$ & $5.52_{-0.48}^{+0.51}$ & 0.57 & 74 & 2.55 & 7.70 \\
2 & 0.71 & $0.32_{-0.21}^{+0.21}$ & $7.17_{-1.18}^{+1.61}$ & $6.57_{-0.51}^{+0.57}$ & 0.62 & 79 & 2.82 & 8.50 \\
3 & 1.89 & $0.34_{-0.20}^{+0.21}$ & $7.57_{-1.29}^{+1.83}$ & $8.02_{-0.63}^{+0.66}$ & 0.94 & 80 & 3.42 & 10.31 \\
4 & 2.33 & $0.36_{-0.20}^{+0.21}$ & $7.52_{-1.21}^{+1.64}$ & $7.99_{-0.57}^{+0.63}$ & 0.70 & 84 & 3.42 & 10.33 \\
5 & 3.61 & $0.26_{-0.18}^{+0.18}$ & $6.29_{-0.93}^{+1.22}$ & $8.08_{-0.60}^{+0.66}$ & 0.70 & 80 & 3.13 & 9.43 \\
6 & 4.58 & $0.27_{-0.17}^{+0.18}$ & $5.93_{-0.84}^{+1.09}$ & $7.90_{-0.57}^{+0.66}$ & 0.61 & 83 & 3.00 & 9.04 \\
7 & 5.56 & $0.24_{-0.17}^{+0.17}$ & $6.14_{-0.87}^{+1.12}$ & $7.66_{-0.57}^{+0.60}$ & 0.76 & 83 & 2.93 & 8.84 \\
8 & 6.89 & $0.28_{-0.15}^{+0.16}$ & $5.88_{-0.75}^{+0.95}$ & $6.91_{-0.48}^{+0.51}$ & 0.78 & 92 & 2.67 & 8.05 \\
9 & 8.50 & $0.22_{-0.15}^{+0.17}$ & $5.25_{-0.68}^{+0.86}$ & $6.67_{-0.51}^{+0.54}$ & 0.88 & 85 & 2.40 & 7.24 \\
10 & 10.11 & $0.25_{-0.17}^{+0.19}$ & $4.86_{-0.64}^{+0.83}$ & $5.82_{-0.51}^{+0.51}$ & 0.83 & 77 & 2.08 & 6.27 \\
11 & 12.14 & $0.18_{-0.16}^{+0.18}$ & $4.61_{-0.62}^{+0.78}$ & $4.89_{-0.45}^{+0.45}$ & 0.93 & 80 & 1.73 & 5.21 \\
12 & 14.14 & $0.17_{-0.16}^{+0.19}$ & $4.09_{-0.51}^{+0.65}$ & $3.59_{-0.33}^{+0.36}$ & 0.70 & 82 & 1.30 & 3.92 \\
13 & 18.11 & $0.19_{-0.19}^{+0.23}$ & $3.65_{-0.49}^{+0.63}$ & $1.72_{-0.18}^{+0.21}$ & 0.80 & 94 & 0.82 & 2.46 \\
14 & 23.75 & $0.20^{c}$ & $2.12_{-0.95}^{+1.22}$ & $0.15_{-0.09}^{+0.09}$ & 0.75 & 80 & 0.46 & 1.38 \\
\hline
\end{tabular}

${ }^{a}$ Central time of each bin computed from the beginning of the observation.

${ }^{b} F_{\mathrm{x}}$ and $L_{\mathrm{x}}$ are computed in the $2-10 \mathrm{keV}$ band.

${ }^{c} Z / Z_{\odot}$ was kept fixed since it was not constrained by the fit.

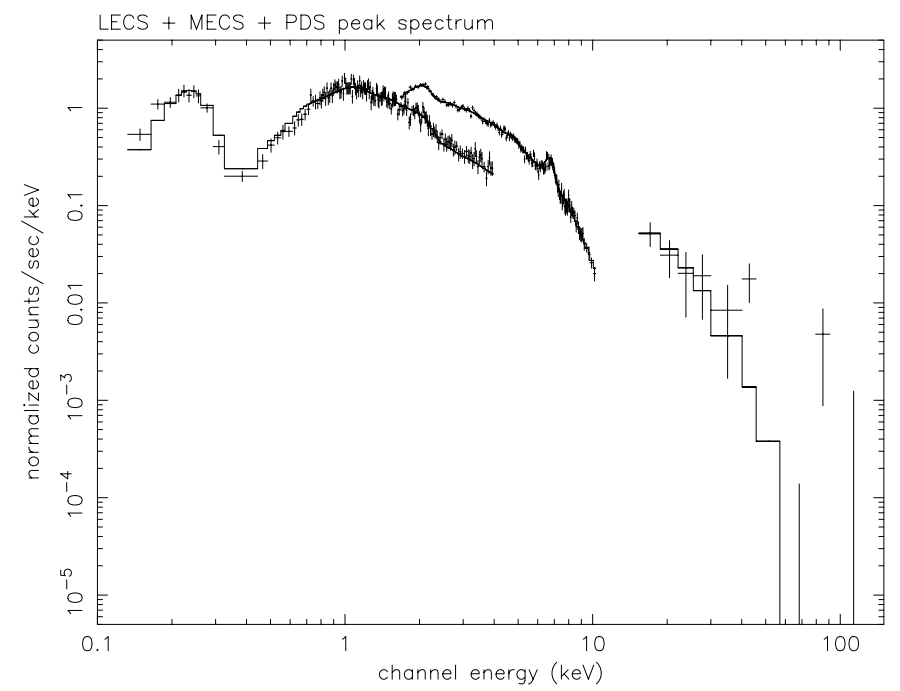

Fig. 4. Overall LECS+MECS+PDS spectrum of the Aug. 1997 flare integrated over the first $24 \mathrm{ksec}$ of the observation. The two-temperature thermal model that best-fits the LECS+MECS data alone is also shown.

Fig. 5. The peak temperature of $\sim 100 \mathrm{MK}$ is reached during the rise phase, before the peak of the emission measure, as commonly observed in solar and stellar flares. The peak emission measure is $E M \sim 8 \times 10^{54} \mathrm{~cm}^{-3}$. The X-ray luminosity in the $2-10 \mathrm{keV}$ band is $L_{\mathrm{X}} \simeq 1.0 \times 10^{32} \mathrm{erg} \mathrm{s}^{-1}$ at the flare peak, and decreases to $1.4 \times 10^{31} \mathrm{erg} \mathrm{s}^{-1}$ at the end of the flare. By integrating $L_{\mathrm{X}}$ over the duration of the flare we find that the total energy released during the flare is $E \gtrsim 5 \times 10^{36} \mathrm{erg}$.

We note that there is no significant variation of the metallicity during the flare: the values at the flare peak are a factor $\sim 2$ higher than in quiescence, but, given the large statistical uncertainties of our results, they are practically within the errors from the quiescent values. If we combine together the intervals Nos. 2-4 around the flare peak, we find $Z / Z_{\odot}=0.33_{-0.12}^{+0.13}$, which is still compatible, within the errors, with the quiescent values. If a variation of $Z$ occurred, it was less than a factor $\sim 3$ at the $90 \%$ confidence level. The lack of a significant increase in the metallicity is in contrast with the $A S C A$ observations of UX Ari by Güdel et al. (1999) and with the BeppoSAX observation of Algol by Favata \& Schmitt (1999), who found enhancements of the metal abundances of, respectively, a factor $\sim 5$ and 10 at the flare peak with respect to the quiescent level. Our results are consistent however with the factor 3 upper limit found by Maggio et al. (2000) for variations of $Z$ during large flares on AB Dor observed with BeppoSAX. We cannot exclude that the abundances of individual elements changed in non-solar proportion during the flare, but this cannot be tested with BeppoSAX due to its lower 


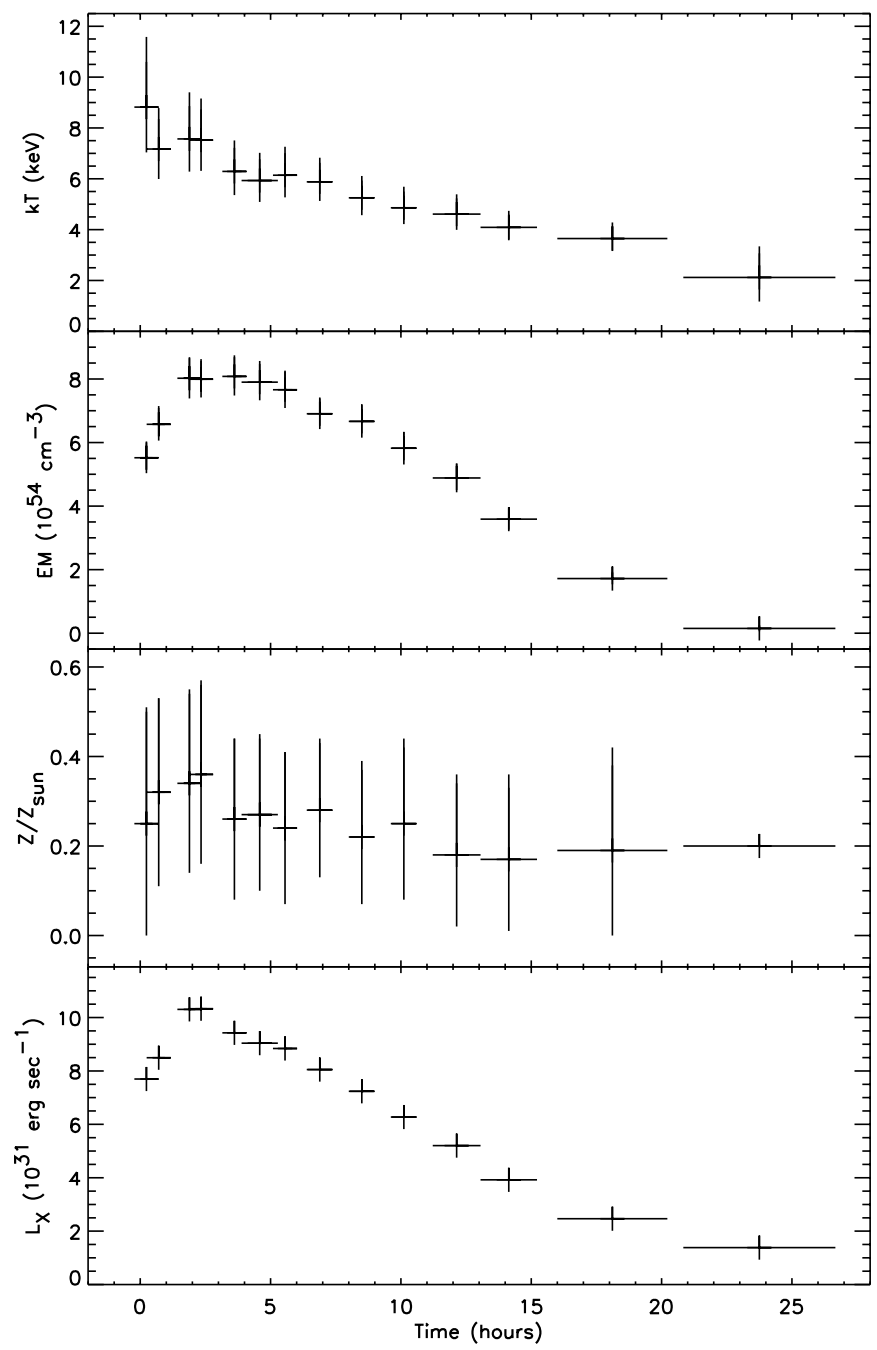

Fig. 5. Time evolution of the temperature, emission measure, metallicity and X-ray luminosity during the Aug. 1997 flare.

spectral resolution (with respect to $A S C A / \mathrm{SIS}$ ) that allows deriving only the global metallicity $Z$ in a reliable way.

\section{Analysis of the flare evolution}

We have analysed the flare evolution by using the tworibbon (2-R) flare model developed by Kopp \& Poletto (1984) for the solar case and extended to stellar flares by Poletto et al. (1988). This model assumes that a disruptive event opens an arcade of loops; the open field lines are then driven towards a neutral sheet, where they reconnect at progressively higher altitudes, forming a growing system of loops. The magnetic energy released in the reconnection process provides the continuous heating responsible for the $\mathrm{X}$-ray emission during the flare. A modified version of the model was applied by Güdel et al. (1999) to the rise phase of the UX Ari flare observed by $A S C A$.

The model assumes that, along meridional planes, closed field lines below the neutral point are described by Legendre polynomials $P_{n}$ of order $n$, while above the neutral point the magnetic field is radial. A magnetic arcade of loops is composed by one lobe of $P_{n}$, axisymmetrically extended in longitude along the East-West direction. The degree of the Legendre polynomial is linked to the size of the magnetic region: higher values of $n$ imply smaller regions.

The rate of magnetic energy release per radian in longitude is given by (Poletto et al. 1988):

$$
\begin{aligned}
\frac{\mathrm{d} E}{\mathrm{~d} t}=\frac{1}{8 \pi} 2 n(n+1)(2 n+ & 1)^{2} R_{*}^{3} B_{\mathrm{m}}^{2}\left[I_{12}(n) / P_{n}^{2}\left(\theta_{1,2}\right)\right] \\
& \times \frac{y^{2 n}\left(y^{2 n+1}-1\right)}{\left[n+(n+1) y^{2 n+1}\right]^{3}} \frac{\mathrm{d} y}{\mathrm{~d} t},
\end{aligned}
$$

where $B_{\mathrm{m}}$ is the maximum surface magnetic field in the region, $\theta_{1,2}$ are the colatitudes of the lobe boundaries, $I_{12}=\int P_{n}^{2}(\theta) \mathrm{d}(\cos \theta)$, where the integral is evaluated between the lobe boundaries, and $y$ is the position of the neutral point in units of the stellar radius $R_{*}$. The upward motion of the neutral point is described by:

$y=1+\left(H_{\mathrm{m}} / R_{*}\right)\left[1-\exp \left(-t / t_{\circ}\right)\right]$,

where $H_{\mathrm{m}}$ is the maximum height reached by the neutral point during the reconnection event, and $t_{\circ}$ is the characteristic time constant of the process. $H_{\mathrm{m}}$ is assumed to be equal to the latitudinal width of the lobes (Poletto et al. 1988). The loop arcade is assumed to have a longitudinal extent of $1.5 H_{\mathrm{m}}$, as typically observed in solar 2-R flares.

As shown by Poletto et al. (1988), the time profile of the energy release curve is almost exclusively determined by $t_{\circ}$ and it is practically independent of $n$; it is therefore possible to determine uniquely $t_{\circ}$ from the comparison of the model with the observed light curve. On the other hand, the normalization depends on both $n$ and $B_{\mathrm{m}}$, therefore different combinations of these two parameters can reproduce equally well the same observed light curve. Larger $n$ values (i.e. smaller loops) imply higher $B_{\mathrm{m}}$ and vice versa.

In general, the radiation emitted in X-rays will be only a fraction $q$ (assumed constant throughout the flare) of the total magnetic energy released during the flare. In fact, we must take into account radiative losses that fall outside of the observed frequency range; moreover, part of the released magnetic energy might go into accelerated particles or plasma ejections or other mechanical energy losses, instead of being used to heat the plasma. Poletto et al. (1988) assumed that the fraction $q$ was $10 \%$ of the global flare energy release. On the other hand, in the case of a solar flare, Kopp \& Poletto (1984) found that the thermal energy needed to reproduce the observations was only a fraction of 0.003 of the total released energy. Since the value of $q$ is not known a priori, instead of fixing it to an arbitrary value we keep it as a free parameter. The value of $q$ affects the normalization factor, which is now proportional to $q B_{\mathrm{m}}^{2}$; this implies that, for any given $n, B_{\mathrm{m}}$ and $q$ cannot be determined independently in the absence of further constraints.

We have fitted the flare profile using different values of $n$, i.e. $n=3,5,9$ and 17 , corresponding to regions of 
Table 3. Parameters of the 2-R flare model. The time constant $t_{\circ}$ is equal to $110000 \mathrm{~s}$ for all values of $n . V$ is the volume of the arcade and $N_{\mathrm{e}}$ is the electron density, computed at the peak and at the end of the flare.

\begin{tabular}{lllll}
\hline$n$ & 3 & 5 & 9 & 17 \\
\hline Region width & $53^{\circ}$ & $33^{\circ}$ & $20^{\circ}$ & $10^{\circ}$ \\
$H_{\mathrm{m}} / R_{*}$ & 0.93 & 0.58 & 0.33 & 0.18 \\
$q^{1 / 2} B_{\mathrm{m}}(\mathrm{G})$ & 79 & 117 & 193 & 346 \\
$V_{\text {(peak) }}\left(\mathrm{cm}^{3}\right)$ & $2 \times 10^{33}$ & $6 \times 10^{32}$ & $1 \times 10^{32}$ & $2 \times 10^{31}$ \\
$V_{\text {(end) }}\left(\mathrm{cm}^{3}\right)$ & $3 \times 10^{34}$ & $8 \times 10^{33}$ & $1 \times 10^{33}$ & $2 \times 10^{32}$ \\
$N_{\text {e(peak) }}\left(\mathrm{cm}^{-3}\right)$ & $6 \times 10^{10}$ & $1 \times 10^{11}$ & $3 \times 10^{11}$ & $6 \times 10^{11}$ \\
$N_{\text {e(end) }}\left(\mathrm{cm}^{-3}\right)$ & $2 \times 10^{9}$ & $5 \times 10^{9}$ & $1 \times 10^{10}$ & $3 \times 10^{10}$ \\
\hline
\end{tabular}

width $53^{\circ}, 33^{\circ}, 20^{\circ}$ and $10^{\circ}$, respectively, in agreement with typical sizes of spotted areas inferred from optical observations of RS CVn stars (e.g. Vogt \& Hatzes 1991; Donati et al. 1992; Hatzes 1993, 1995). The fit parameters for each $n$ are given in Table 3 , and the resulting curves are shown in Fig. 6. The long duration of the flare decay requires $t_{\circ} \simeq 110000 \mathrm{~s} \sim 30.5$ hours. All curves reproduce quite well the observed flare profile, except for the points around the flare peak. The product $q^{1 / 2} B_{\mathrm{m}}$ ranges from $79 \mathrm{G}$ for $n=3$ to $346 \mathrm{G}$ for $n=17$. We can estimate the electron density in the flaring region by approximating the arcade with a semi-cylinder as in Poletto et al. (1988). We find that, at the flare peak, the electron density ranges from $6 \times 10^{10} \mathrm{~cm}^{-3}$ for $n=3$ to $6 \times 10^{11} \mathrm{~cm}^{-3}$ for $n=17$; at the end of the flare these densities have decreased to $2.5 \times 10^{9}$ and $3 \times 10^{10} \mathrm{~cm}^{-3}$, respectively. We will discuss the physical implications of these results in the following section.

\section{Discussion and conclusions}

In this paper we have presented the results of two BeppoSAX observations of the active binary UX Ari. During the first observation a large, long-duration flare occurred, which reached a peak temperature of $\sim 100 \mathrm{MK}$ and a peak X-ray luminosity of $1 \times 10^{32} \mathrm{erg} \mathrm{s}^{-1}$, and released an energy $>5 \times 10^{36} \mathrm{erg}$. This flare resembles closely the large flare observed 10 years earlier on UX Ari by Ginga (Tsuru et al. 1989): the Ginga flare in fact had a similar decay time, a peak luminosity of $2 \times 10^{32} \mathrm{erg} \mathrm{s}^{-1}$, a peak temperature of $80 \mathrm{MK}$, and released $>10^{37} \mathrm{erg}$. The peak luminosity and temperature are also similar to those reached during the flare observed by $A S C A$ (Güdel et al. 1999), for which however only the rise phase up to the peak was observed.

We have successfully modeled the flare evolution using a two-ribbon flare model. The agreement of the predicted light curve with the observed one is very good, except close to the peak, but the size of the flaring region cannot be uniquely determined from the model unless additional information are available. The total magnetic energy released in the reconnection process depends in fact on both the magnetic field strength $B_{\mathrm{m}}$ and the size (order $n$ ) of

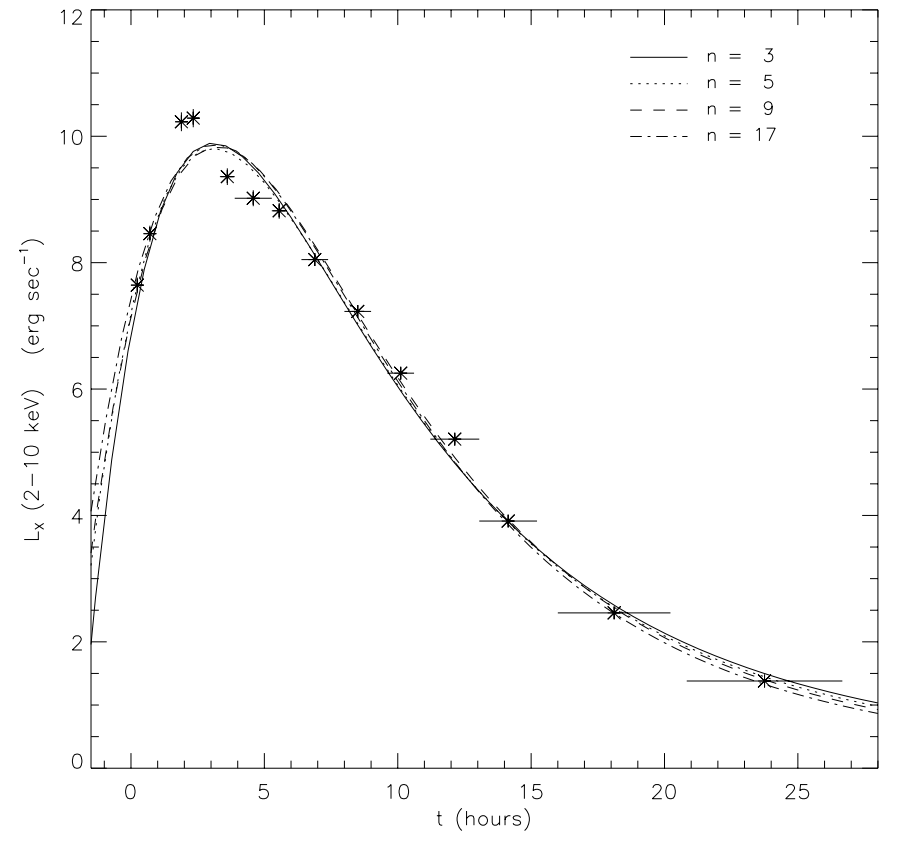

Fig. 6. Comparison of the flare evolution with the results of the 2-R flare model. The curves have been computed for $n=3$, 5,9 , and 17 , corresponding to region sizes of $53^{\circ}, 33^{\circ}, 20^{\circ}$ and $10^{\circ}$, respectively. The characteristic time constant is $\sim 110000 \mathrm{~s}$ for all curves.

the flaring region. For a given $n$, the maximum surface magnetic field strength in the region depends in turn on the fraction $q$ of released energy that is radiated in the $\mathrm{X}$-ray band, which is not known a priori. It is possible to put constraints on the model by requiring that the emitting plasma is confined by the magnetic field. Such a constraint was used by Güdel et al. (1999) in their application of the 2-R model to the $A S C A$ flare: they found an acceptable solution for $n=2$, with $q=0.003$ and $B_{\mathrm{m}}=2600 \mathrm{G}$.

For our flare, we can compute for a given $n$, as a function of time, the minimum magnetic field strength required at the loop top to confine the plasma. Following Güdel et al. (1999) we assume that, at time $t$, the newlyformed X-ray emitting loop below the neutral point has a height

$H_{\mathrm{X}}(t) \simeq H(t)\left[1-\frac{\pi}{2} \frac{v(t)}{v_{\text {evap }}}\right]$

where $H=R_{*}(y-1), v(t)=R_{*} \mathrm{~d} y / \mathrm{d} t$, and $v_{\text {evap }} \simeq$ $500 \mathrm{~km} \mathrm{~s}^{-1}$ is the velocity of evaporation of the heated material from the chromosphere into the loop. Using Eqs. (1) and (2) of Poletto et al. (1988) we can then extrapolate the field back to the photosphere at any given time, and derive the minimum value of $B_{\mathrm{m}}$ which allows the confinement of the plasma for the whole duration of the flare. We find that the required surface field is $B_{\mathrm{m}}=1400,3800,11600$ and $36000 \mathrm{G}$ for $n=3,5,9$ and 17 , respectively. The latter two values are clearly too high. Measurements of magnetic fields on RS CVn stars (e.g. Giampapa et al. 1983; Donati et al. 1992; Donati 1999) indicate that 
magnetic regions have typical surface field strengths of the order of $1 \mathrm{kG}$. The values of $B_{\mathrm{m}}$ for $n=3$ and 5 are consistent with the observed magnetic fields; from Table 6 we derive $q=0.003$ and $q=0.001$, respectively. We therefore conclude that the flare probably took place in a region of size $33^{\circ}-53^{\circ}$, i.e. $\sim 0.6-0.9 R_{*}$ wide, with loop semilength increasing from $\sim 0.1-0.2 R_{*}$ at the flare peak to $\sim 0.5-0.8 R_{*}$ at the end of the flare; the energy emitted in X-rays was only $0.1-0.3 \%$ of the global magnetic energy released in the reconnection process. These results are consistent with those obtained by Güdel et al. (1999) for the $A S C A$ flare.

The high temperatures $\left(\sim 10^{8} \mathrm{~K}\right)$ reached during the flare are responsible for the hard X-ray emission up to $40-50 \mathrm{keV}$ detected in the PDS instrument. Hard X-ray emission has been detected by BeppoSAX also on Algol (Favata \& Schmitt 1999) and AB Dor (Pallavicini \& Tagliaferri 1999; Pallavicini 2001). In all cases, hard $\mathrm{X}$-rays have been observed only during the rise phase and at the peak of large flares, when temperatures $\gtrsim 10^{8}$ are reached, and can be attributed to the thermal emission from such hot plasma, with no need for an additional non-thermal component. This is in contrast with the solar case, where hard X-ray emission above $10 \mathrm{keV}$, usually observed during the impulsive phase of flares, is attributed to non-thermal bremsstrahlung of electrons accelerated during the flare energy release and precipitating into the dense chromospheric layers at the base of the flaring loops. However, the solar non-thermal hard Xray emission is only a tiny fraction $\left(\sim 10^{-5}\right)$ of the soft X-ray flux, while the thermal hard X-ray tail observed in the stellar case is much stronger: for UX Ari we find that the ratio between the hard $(>10 \mathrm{keV})$ and the soft $(0.1-10 \mathrm{keV}) \mathrm{X}$-ray flux is $\sim 0.2$ at the flare peak. If the star also produces a fraction of $10^{-5}$ in non-thermal hard $\mathrm{X}$-rays, it would require extremely high sensitivity or effective area at higher energies to get a significant detection of such non-thermal emission, given the much stronger thermal component. Alternatively, the star could produce a much larger fraction of non-thermal emission, comparable to the high-energy tail of the thermal spectrum (note that the plasma temperature at the flare peak observed in these large stellar flares is much higher than the typical temperature of $\sim 20-40 \times 10^{6} \mathrm{~K}$ observed in solar flares). This cannot be neither excluded nor proved with the data at hand (see Pallavicini 2001 for a discussion of this point in relation to large flares observed on $\mathrm{AB}$ Dor).

We find that the coronal abundance is strongly subsolar $\left(Z=0.2-0.3 Z_{\odot}\right)$, confirming the results obtained with $A S C A$ (Güdel et al. 1999). Although, contrary to $A S C A$, BeppoSAX is not able to constrain individual abundances because of its lower spectral resolution in comparison with the ASCA/SIS, our global metallicity $Z$ agrees well with the individual quiescent abundances derived with $A S C A$, that were in the range $0.17-0.43$, with the only exception of $\mathrm{Ni}$ and $\mathrm{Ne}$. The $A S C A$ observation showed also a significant increase of the abundances during the flare, up to a factor 5 for iron.
Abundance variations during flares have been found also in other stars, such as Algol (Ottmann \& Schmitt 1996; Favata \& Schmitt 1999), II Peg (Mewe et al. 1997), EV Lac (Favata et al. 2000), YY Gem (Tagliaferri et al. 2001). However, in our case no significant variation of the metallicity of UX Ari during the flare with respect to the quiescent value was observed: if any increase occurred, it was less than a factor of $\sim 3$.

Subsolar coronal abundances are commonly found for the most active stars. While this might simply reflect a lower metallicity of the star, in some cases, such as AB Dor (Mewe et al. 1996; Maggio et al. 2000; Güdel et al. 2001), or II Peg (Covino et al. 2000), the derived coronal metallicity was found to be significantly lower than the measured photospheric value. Unfortunately, to our knowledge, the photospheric abundance of UX Ari is not known, so we cannot test whether its coronal abundance is lower than the photospheric one or not.

We have shown that, using the new LECS response matrices released in January 2000, we are able to fit the quiescent spectrum with a value of the interstellar hydrogen column density $N_{\mathrm{H}}$ very close to the expected one, and nearly one order of magnitude lower than that obtained with the old matrices. Our finding therefore seems to confirm the suggestion (Tagliaferri et al. 1999; Pallavicini 2001) that the high $N_{\mathrm{H}}$ found in all previous BeppoSAX observations of stellar coronae was most likely due to calibration problems at low energies. We find however that the absorption is much higher (by a factor $\sim 5$ ) during the whole flare, remaining constant up to the flare end. A strong increase in the interstellar absorption (by a factor $>10$ ) was also observed during the Algol flare (Favata \& Schmitt 1999). A possible explanation for the increased absorption is the occurrence of a coronal mass ejection at the flare onset, which could have provided the absorbing material along the line of sight during the whole flare. On the Sun, coronal mass ejections may be accompanied by soft X-ray flares, that are generally long-duration events taking place in arcade structures. Therefore, the occurrence of a coronal mass ejection would be consistent with our interpretation of the flare in terms of a $2-\mathrm{R}$ model.

Acknowledgements. We thank the BeppoSAX Science Data Center (SDC) for their support in the data analysis. We also thank Dr. M. Güdel for his comments as referee of this paper. This research was financially supported by Ministero dell'Università e della Ricerca Scientifica e Tecnologica (MURST) and by the Italian Space Agency (ASI).

\section{References}

Covino, S., Tagliaferri, G., Pallavicini, R., Mewe, R., \& Poretti, E. 2000, A\&A, 355, 681

Dempsey, R. C., Linsky, J. L., Fleming, T. A., \& Schmitt, J. H. M. M. 1993, ApJS, 86, 599

Dempsey, R. C., Linsky, J. L., Schmitt, J. H. M. M., \& Fleming, T. A. 1993, ApJ, 413, 333

Donati, J.-F. 1999, MNRAS, 302, 457

Donati, J.-F., Brown, S. F., Semel, M., et al. 1992, A\&A, 265, 682 
ESA 1997, The Hipparcos and Tycho Catalogues, ESA SP-1200 Favata, F., Mineo, T., Parmar, A. N., \& Cusumano, G. 1997, A\&A, 324, L41

Favata, F., \& Schmitt, J. H. M. M. 1999, A\&A, 350, 900

Favata, F., Reale, F., Micela, G., et al. 2000, A\&A, 353, 987

Fiore, F., Guainazzi, M., \& Grandi, P. 1999, Cookbook for NFI BeppoSAX Spectral Analysis v. 1.2, available at www.sdc.asi.it

Franciosini, E., Pallavicini, R., Bastian, T., et al. 2001, in ASP Conf. Ser., 223, Cool Stars, Stellar Systems, and the Sun, 11th Cambridge Workshop, ed. R. J. Garcia Lopez, et al. (San Francisco: ASP), CD-930

Gehrels, N. 1986, ApJ, 303, 336

Giampapa, M. S., Golub, L., \& Worden, S. P. 1983, ApJ, 268, L121

Güdel, M., Linsky, J. L., Brown, A., \& Nagase, F. 1999, ApJ, 511,405

Güdel, M., Audard, M., Briggs, K., et al. 2001, A\&A, 365, L336

Hatzes, A. P. 1993, ApJ, 410, 777

Hatzes, A. P. 1995, AJ, 109, 350

Kopp, R. A., \& Poletto, G. 1984, Sol. Phys., 93, 351

Maggio, A., Pallavicini, R., Reale, F., \& Tagliaferri, G. 2000, A\&A, 356, 627

Mewe, R., Kaastra, J. S., \& Liedahl, D. A. 1995, Legacy, 6, 16

Mewe, R., Kaastra, J., van den Oord, G., Vink, J., \& Tawara, Y. 1997, A\&A, 320, 147
Mewe, R., Kaastra, J. S., White, S. M., \& Pallavicini, R. 1996, A\&A, 315, 170

Ottmann, R., \& Schmitt, J. H. M. M. 1996, A\&A, 307, 813

Pallavicini, R. 2001, in ASP Conf. Ser. 223, Cool Stars, Stellar Systems, and the Sun, 11th Cambridge Workshop, ed. R. J. Garcia Lopez, et al. (San Francisco: ASP), 377

Pallavicini, R., \& Tagliaferri, G. 1999, in Highlights in X-ray Astronomy, ed. B. Aschenbach, \& M. J. Freyberg, MPE Report, 272, 378

Paresce, F. 1984, AJ, 89, 1022

Poletto, G., Pallavicini, R., \& Kopp, R. A. 1988, A\&A, 201, 93

Rodonò, M., Pagano, I., Leto, G., et al. 1999, A\&A, 346, 811

Tagliaferri, G., Covino, S., Cutispoto, G., \& Pallavicini, R. 1999, A\&A, 345, 514

Tagliaferri, G., Covino, S., Panzera, M. R., Pallavicini, R., \& Schmitt, J. H. M. M. 2001, in ASP Conf. Ser., 223, Cool Stars, Stellar Systems, and the Sun, 11th Cambridge Workshop, ed. R. J. Garcia Lopez, et al. (San Francisco: ASP), CD-1177

Tsuru, T., Makishima, K., Ohashi, T., et al. 1989, PASJ, 41, 679

Walter, F., Charles, P., \& Bowyer, S. 1978, ApJ, 225, L119

Vogt, S. S., \& Hatzes, A. P. 1991, in IAU Coll. 130, The Sun and Cool Stars: Activity, Magnetism, Dynamos, ed. I. Tuominen, et al. (Berlin: Springer), 297 